\title{
Pathogenic Variability and Fungicidal Sensitivity of Fusarium spp. Causing Wilt in Chilli in Karnataka
}

\author{
S. Deepthi Gowda ${ }^{*}$, S. Amruta Bhat, V. Kantharaju, Sandhyarani Nishani, \\ Abdul Kareem and H. P. Hadimani \\ Department of Plant Pathology, Kittur Rani Channamma College of Horticulture, \\ Arabhavi- 591218, Karnataka, India \\ *Corresponding author
}

A B S T R A C T

\section{Keywords \\ Fusarium solani, Fusarium oxysporum, Chilli, Pathogenic variability and Fungicidal sensitivity}

\section{Article Info}

Accepted:

04 September 2020 Available Online: 10 October 2020

\section{Introduction}

Chilli (Capsicum annuum L.) belonging to family solanaceae is a important spice and vegetable crop. Chillies are good source of vitamin $\mathrm{A}$, vitamin $\mathrm{C}$, vitamin $\mathrm{E}$, folic acid, calcium, potassium and antioxidants like flavonoids, capsaicinoids and carotenoids. Several varieties of chilli are cultivated for varied uses like vegetable, pickles, spice and condiments. It is being used for imparting taste, flavour, and colour to food and also used in preservative, pharmaceutical, perfumery, cosmetic products and religious rituals. Universally, major chilli producing countries are China, Mexico, Turkey, Indonesia, India, Spain and United States. India is one of the largest producers and exporters of this crop and ranks second among world's chilli exporting countries.

In India, area under green chilli accounts to 2.87 lakh ha with a production of 34.06 lakh metric tonnes and that under red chilli is 8.11 
lakh ha with a production of 15.20 lakh metric tonnes. Within the country, the leading chilli producing states are Andhra Pradesh, Telangana, Maharastra, Karnataka, Madhya Pradesh, West Bengal and Tamil Nadu. Karnataka accounts to 15 per cent share of the total green chilli production in the country with an area of 0.45 lakh ha and production of 6.07 lakh tonnes. In Karnataka, the crop is mainly cultivated in districts like Dharwad, Haveri, Koppal, Bellary, Raichur, Kalburgi and Belagavi (Anon., 2017). Chilli production in the country is increasingly constrained by several abiotic and biotic factors.

Among the biotic factors, diseases caused by fungi, bacteria and viruses are responsible for drastic reduction of yield and quality of chilli. In recent years, wilt caused by the fungus Fusarium spp. has emerged as a serious disease in chilli. Incidence of this disease has been reported to vary from 2 to 85 per cent in different chilli growing regions of India (Anon., 2005).

The yield loss due to the disease is known to vary from 10 to 80 per cent worldwide (Loganathan et al., 2013), 5 to 57 per cent in South India (Raghu et al., 2014) and 20 per cent in black cotton soils of northern Karnataka (Devika Rani et al., 2007). Fusarium spp. are soil borne in nature and produce chlamydospores which enables the fungus to persist in soil for long time even in the absence of host plant. Hence, once soil is infested with the pathogenic Fusarium, it becomes difficult to cultivate susceptible varieties and lot of fungicides need to be drenched to manage the disease. Fusarium spp. exhibit lot of pathogenic, variability and also varied sensitivity to different fungicides. Hence, the present study was aimed to study both these aspects to understand the pathogenic variability of Fusarium spp. collected from different infested chilli fields and their responses towards tested fungicides.

\section{Materials and Methods}

\section{Collection, pathogen isolation and identification}

Fifty one isolates of Fusarium spp. were collected from major chilli growing districts of Karnataka namely Bagalkot, Bellary, Belagavi, Haveri, Raichur and Tumkur. For isolation of the pathogen, the roots and basal stem portion of completely and partially wilted chilli plants were cut into small pieces of 1-2 cm size and surface-sterilized with sodium hypochlorite solution for one to two minute and washed repeatedly in sterilized water and placed in Petriplates containing PDA medium and incubated at $27 \pm 1^{\circ} \mathrm{C}$. The purified culture of the pathogen was obtained by hyphal tip method and maintained at $4{ }^{\circ} \mathrm{C}$ temperature and used for further studies. The pathogen isolates were mainly identified on the basis of cultural and morphological characters of Fusarium as described by Leslie and Summerell (2006).

\section{Pathogenic variability}

To study pathogenic variability of different isolates, cultivar of chilli Byadagi Dabbi was inoculated by root dip technique. Twenty five days old seedlings of Fusarium susceptible chilli variety Byadagi Dabbi were taken for the study. Briefly, the roots of the seedlings were thoroughly washed in tap water followed by sterile water and trimmed at root hair region. Then the seedlings were inoculated by dipping the roots for $30 \mathrm{~min}$ in spore suspension of fungus (at the rate of $1 \times 106$ spores/ml) which was cultured on PDB for ten days.

Inoculated seedlings were transferred to pots containing sterilized soil and maintained in poly house. Set of seedlings without inoculation was maintained as control. Symptom expression was recorded regularly 
from third day of inoculation upto 3 weeks. Disease was scored according to scale, 1- No symptoms, 2- Slight chlorosis, wilting or stunting of the plant, 3-Moderate chlorosis, wilting or stunting of the plant, 4- Severe chlorosis, wilting or stunting of the plant, 5Death of the plant as described by Nirmaladevi and Srinivas (2012).

\section{Sensitivity to fungicides}

Different isolates of fusarium were tested for their sensitivity to fungicides viz., Mancozeb (contact), Carbendezim and Propiconazole (systemic) at concentration $0.25,0.2$ and 0.05 per cent respectively. Poisoned food technique was adopted to test the sensitivity. Required quantity of individual fungicide was added separately into molten and cooled potato dextrose agar so as to get the desired concentration of fungicides. Later $20 \mathrm{ml}$ of the poisoned medium was poured into sterile Petriplates. Mycelial discs of $5 \mathrm{~mm}$ size from actively growing culture of the fungus were cut out by a sterile cork borer and one such disc was placed at the center of each agar plate.

Control was maintained without adding any fungicides to the medium. Each treatment was replicated twice. Then such plates were incubated at room temperature for ten days and radial colony growth was measured. The efficacy of a fungicide was expressed as per cent inhibition of mycelial growth over control that was calculated by using the formula suggested by Vincent (1947).



Where,

$\mathrm{I}=$ Per cent inhibition

$\mathrm{C}=$ Radial growth in control $(\mathrm{mm})$

$\mathrm{T}=$ Radial growth in treatment $(\mathrm{mm})$

\section{Results and Discussion}

\section{Isolation, purification and identification of pathogen}

A total of fifty one isolates of fungus were isolated from chilli plant samples collected from different places by standard tissue isolation procedure and they were purified and maintained on PDA as described in material and methods. The fungal isolates were identified by comparing their morpho-cultural characters like production of spores such as micro, macro and chlamydo-spores, shape, size and septation in macro-conidia and pigmentation in culture with those described by Leslie and Summerell (2006). Out of 51 isolates, three isolates (FO 22, FO 26, FO 30) were identified as $F$. oxysporum and 48 isolates were identified as $F$. solani. Fusarium oxysporum had thin-walled, relatively slender and 3-5 septate macro-conidia, evenly curved fusoid with the widest part in the middle and pointed at both the ends. The micro-conidia were formed on smaller false heads with floccose, sparse or abundant mycelia, which ranged from white to pale violet in pigmentation. Whereas, Fusarium solani had thick-walled, curved, dorsoventrally straight, relatively wider, stout and robust macro-conidia with the widest diameter in the upper half of the spore. The micro-conidia were monophilidic and formed on relatively longer false heads with white to cream colour pigmentation with sparse mycelium. The chlamydospores were formed singly, in pairs, in chains or in clumps in both the species. Chlamydospores of $F$. solani were smooth textured whereas; in $F$. oxysporum they were rough textured.

\section{Pathogenic variability}

Pathogenic variability of different isolates was tested on susceptible cultivar Byadagi dabbi by root dip technique as explained in material and methods. 
Table.1 Pathogenic variability of Fusarium isolates of chilli on $c v$. Byadagi Dabbi

\begin{tabular}{|c|c|c|c|c|c|c|c|c|}
\hline \multirow{2}{*}{$\begin{array}{l}\text { Isolate } \\
\text { name }\end{array}$} & \multicolumn{4}{|c|}{ Symptoms (DAI) } & \multicolumn{4}{|c|}{ Per cent wilt incidence (DAI) } \\
\hline & $3^{\text {rd }}$ & $7^{\text {th }}$ & $15^{\text {th }}$ & $22^{\text {nd }}$ & $3^{\text {rd }}$ & $7^{\text {th }}$ & $15^{\text {th }}$ & $22^{\text {nd }}$ \\
\hline FS 1 & $\mathrm{CL}$ & $\mathrm{CL}$ and $\mathrm{MW}$ & SW & SW & - & - & - & - \\
\hline FS 2 & $\mathrm{CL}$ & $\mathrm{CL}$ and $\mathrm{MW}$ & SW & SW & - & - & 100 & - \\
\hline FS 3 & $\mathrm{CL}$ & CL and MW & SW & SW & - & - & 100 & - \\
\hline FS 4 & $\mathrm{CL}$ & $\mathrm{CL}$ and $\mathrm{MW}$ & SW & SW & - & - & 100 & - \\
\hline FS 5 & $\mathrm{CL}$ & $\mathrm{CL}$ and $\mathrm{MW}$ & SW & SW & - & - & 100 & - \\
\hline FS 6 & $\mathrm{CL}$ & $\mathrm{CL}$ and $\mathrm{MW}$ & SW & SW & - & - & 100 & - \\
\hline FS 7 & $\mathrm{CL}$ & $\mathrm{CL}$ and $\mathrm{MW}$ & SW & SW & - & - & 100 & - \\
\hline FS 8 & $\mathrm{CL}$ & $\mathrm{CL}$ and $\mathrm{MW}$ & SW & SW & - & - & 100 & - \\
\hline FS 9 & $\mathrm{CL}$ & SW & SW & SW & - & 100 & - & - \\
\hline FS 10 & $\mathrm{CL}$ & SW & SW & SW & - & 100 & - & - \\
\hline FS 11 & $\mathrm{CL}$ & SW & SW & SW & - & 100 & - & - \\
\hline FS 12 & $\mathrm{CL}$ & SW & SW & SW & - & 100 & - & - \\
\hline FS 13 & $\mathrm{CL}$ & SW & SW & SW & - & 100 & - & - \\
\hline FS 14 & CL & SW & SW & SW & - & 100 & - & - \\
\hline FS 15 & $\mathrm{CL}$ & SW & SW & SW & - & 100 & - & - \\
\hline FS 16 & $\mathrm{CL}$ & SW & SW & SW & - & 100 & - & - \\
\hline FS 17 & CL & CL & SW & SW & - & - & 100 & - \\
\hline FS 18 & $\mathrm{CL}$ & CL & SW & SW & - & - & 100 & - \\
\hline FS 19 & $\mathrm{CL}$ & SW & SW & SW & - & 100 & - & - \\
\hline FS 20 & $\mathrm{CL}$ & $\mathrm{CL}$ & $\mathrm{CL}$ & $\mathrm{CL}$ & - & - & - & - \\
\hline FS 21 & $\mathrm{CL}$ & SW & SW & SW & - & 100 & - & - \\
\hline FO 22 & $\mathrm{CL}$ & SW & SW & SW & - & 100 & - & - \\
\hline FS 23 & $\mathrm{CL}$ & CL & SW & SW & - & - & 100 & - \\
\hline FS 24 & $\mathrm{CL}$ & SW & SW & SW & - & 100 & - & - \\
\hline FS 25 & $\mathrm{CL}$ & CL & $\mathrm{CL}$ & $\mathrm{CL}$ & - & - & 100 & - \\
\hline FO 26 & $\mathrm{CL}$ & SW & SW & SW & - & 100 & - & - \\
\hline FS 27 & $\mathrm{CL}$ & CL & $\mathrm{CL}$ & $\mathrm{CL}$ & - & 100 & - & - \\
\hline FS 28 & $\mathrm{CL}$ & SW & SW & SW & - & 100 & - & - \\
\hline FS 29 & $\mathrm{CL}$ & CL & SW & SW & - & - & 100 & - \\
\hline FO 30 & $\mathrm{CL}$ & SW & SW & SW & - & 100 & - & - \\
\hline FS 31 & CL & CL & SW & SW & - & - & 100 & - \\
\hline FS 32 & CL & CL & SW & SW & - & - & 100 & - \\
\hline FS 33 & $\mathrm{CL}$ & CL & SW & SW & - & - & 100 & - \\
\hline FS 34 & $\mathrm{CL}$ & $\mathrm{CL}$ & SW & SW & - & - & 100 & - \\
\hline FS 35 & $\mathrm{CL}$ & CL & SW & SW & - & - & 100 & - \\
\hline FS 36 & $\mathrm{CL}$ & CL & SW & SW & - & - & 100 & - \\
\hline FS 37 & $\mathrm{CL}$ & CL & SW & SW & - & - & 100 & - \\
\hline FS 38 & $\mathrm{CL}$ & CL & SW & SW & - & - & 100 & - \\
\hline FS 39 & - & - & - & $\mathrm{CL}$ and $\mathrm{W}$ & - & - & - & - \\
\hline FS 40 & & - & SW & SW & - & - & 100 & - \\
\hline FS 41 & - & - & - & $\mathrm{CL}$ and $\mathrm{W}$ & - & - & - & - \\
\hline FS 42 & - & - & - & $\mathrm{CL}$ and $\mathrm{W}$ & - & - & - & - \\
\hline FS 43 & $\mathrm{CL}$ & CL & SW & SW & - & - & 100 & - \\
\hline FS 44 & $\mathrm{CL}$ & CL & SW & SW & - & - & 100 & - \\
\hline FS 45 & $\mathrm{CL}$ & CL & $\mathrm{CL}$ & CL & - & - & - & - \\
\hline FS 46 & $\mathrm{CL}$ & $\mathrm{CL}$ & SW & SW & - & - & 100 & - \\
\hline FS 47 & $\mathrm{CL}$ & CL & $\mathrm{CL}$ & $\mathrm{CL}$ & - & 100 & - & - \\
\hline FS 48 & $\mathrm{CL}$ & CL & $\mathrm{CL}$ & CL & - & 100 & - & - \\
\hline FS 49 & CL & CL & CL & CL & - & 100 & - & - \\
\hline FS 50 & $\mathrm{CL}$ & CL & CL & CL & - & 100 & - & - \\
\hline FS 51 & $\mathrm{CL}$ & CL & $\mathrm{CL}$ & CL & - & 100 & - & - \\
\hline
\end{tabular}


Table.2 Grouping of Fusarium isolates of chilli based on pathogenicity

\begin{tabular}{|c|l|c|}
\hline Virulence grade & \multicolumn{1}{|c|}{ Isolate name } & Number of isolates \\
\hline Highly pathogenic & FS 9, FS 10, FS 11, FS 12, FS 13, FS 14, FS 15, FS & 21 \\
& 16, FS 19, FS 21, FO 22, FS 24, FO 26, FS 27, FS & \\
& 28, FO 30, FS 47, FS 48, FS 49, FS 50, FS 51 & \\
\hline Moderately pathogenic & FS 2, FS 3, FS 4, FS 5, FS 6, FS 7, FS 8, FS 17, FS & 25 \\
& 18, FS 20, FS 23, FS 25, FS 29, FS 31, FS 32, FS & \\
& 33, FS 34, FS 35, FS 36, FS 37, FS 38, FS 40, FS & \\
\hline 43, FS 44, FS 46 & 4 \\
\hline Weakly pathogenic & FS 39, FS 41, FS 42, FS 45 & \\
\hline
\end{tabular}

Table.3 Sensitivity of Fusarium isolates of chilli to three different fungicides

\begin{tabular}{|c|c|c|c|}
\hline \multirow{2}{*}{$\begin{array}{l}\text { Isolate } \\
\text { name }\end{array}$} & \multicolumn{3}{|c|}{ Per cent inhibition of colony growth } \\
\hline & $\begin{array}{c}\text { Mancozeb } \\
(0.25 \%)\end{array}$ & $\begin{array}{c}\text { Carbendezim } \\
(0.2 \%)\end{array}$ & Propiconazole $(0.05 \%)$ \\
\hline FS 1 & $\begin{array}{c}60.00 \\
(50.75)\end{array}$ & $\begin{array}{c}91.11 \\
(72.62)\end{array}$ & $\begin{array}{c}80.56 \\
(63.81)\end{array}$ \\
\hline FS 2 & $\begin{array}{c}76.11 \\
(60.72)\end{array}$ & $\begin{array}{l}99.73 \\
(86.98)\end{array}$ & $\begin{array}{c}82.44 \\
(65.20)\end{array}$ \\
\hline FS 3 & $\begin{array}{c}73.33 \\
(58.90)\end{array}$ & $\begin{array}{c}97.89 \\
(81.61)\end{array}$ & $\begin{array}{c}89.44 \\
(71.09)\end{array}$ \\
\hline FS 4 & $\begin{array}{c}73.33 \\
(58.90)\end{array}$ & $\begin{array}{c}99.71 \\
(86.87)\end{array}$ & $\begin{array}{c}89.11 \\
(70.70)\end{array}$ \\
\hline FS 5 & $\begin{array}{c}73.33 \\
(58.90)\end{array}$ & $\begin{array}{ll} & 95.56 \\
(77.80) & \end{array}$ & $\begin{array}{c}82.44 \\
(65.20)\end{array}$ \\
\hline FS 6 & $\begin{array}{c}60.00 \\
(50.75)\end{array}$ & $\begin{array}{c}93.33 \\
(75.00)\end{array}$ & $\begin{array}{c}80.89 \\
(64.05)\end{array}$ \\
\hline FS 7 & $\begin{array}{c}73.33 \\
(58.90)\end{array}$ & $\begin{array}{c}91.11 \\
(72.62)\end{array}$ & $\begin{array}{c}82.44 \\
(65.20)\end{array}$ \\
\hline FS 8 & $\begin{array}{c}56.11 \\
(48.49)\end{array}$ & $\begin{array}{c}92.22 \\
(73.77)\end{array}$ & $\begin{array}{c}86.78 \\
(68.65)\end{array}$ \\
\hline FS 9 & $\begin{array}{l}100.00 \\
(90.00)\end{array}$ & $\begin{array}{c}91.11 \\
(72.62)\end{array}$ & $\begin{array}{c}80.56 \\
(63.81)\end{array}$ \\
\hline FS 10 & $\begin{array}{l}100.00 \\
(90.00)\end{array}$ & $\begin{array}{c}93.33 \\
(75.00)\end{array}$ & $\begin{array}{c}84.67 \\
(66.92)\end{array}$ \\
\hline FS 11 & $\begin{array}{l}100.00 \\
(90.00)\end{array}$ & $\begin{array}{l}97.78 \\
(81.39)\end{array}$ & $\begin{array}{l}89.11 \\
(70.70)\end{array}$ \\
\hline FS 12 & $\begin{array}{c}56.11 \\
(48.99)\end{array}$ & $\begin{array}{l}95.56 \\
(77.80)\end{array}$ & $\begin{array}{ll} & 82.78 \\
(65.45) & \end{array}$ \\
\hline FS 13 & $\begin{array}{c}61.11 \\
(51.40)\end{array}$ & $\begin{array}{c}99.73 \\
(86.98)\end{array}$ & $\begin{array}{c}84.67 \\
(66.92)\end{array}$ \\
\hline FS 14 & $\begin{array}{c}61.11 \\
(51.40)\end{array}$ & $\begin{array}{l}99.69 \\
(86.77)\end{array}$ & $\begin{array}{l}100.00 \\
(90.00)\end{array}$ \\
\hline FS 15 & $\begin{array}{c}71.22 \\
(57.53)\end{array}$ & $\begin{array}{l}99.64 \\
(86.52)\end{array}$ & $\begin{array}{c}82.44 \\
(65.23) \\
\end{array}$ \\
\hline FS 16 & $\begin{array}{c}47.22 \\
(43.39)\end{array}$ & $\begin{array}{l}91.11 \\
(72.62)\end{array}$ & $\begin{array}{c}87.22 \\
(69.03)\end{array}$ \\
\hline FS 17 & $\begin{array}{c}67.89 \\
(55.46)\end{array}$ & $\begin{array}{l}99.67 \\
(86.67)\end{array}$ & $\begin{array}{l}100.00 \\
(90.00)\end{array}$ \\
\hline
\end{tabular}




\begin{tabular}{|c|c|c|c|}
\hline FS 18 & $\begin{array}{c}75.78 \\
(60.49)\end{array}$ & $\begin{array}{c}99.71 \\
(86.87)\end{array}$ & $\begin{array}{l}100.00 \\
(90.00)\end{array}$ \\
\hline FS 19 & $\begin{array}{c}61.11 \\
(51.40)\end{array}$ & $\begin{array}{c}99.69 \\
(86.77)\end{array}$ & $\begin{array}{c}89.11 \\
(70.70)\end{array}$ \\
\hline FS 20 & $\begin{array}{c}73.56 \\
(59.02)\end{array}$ & $\begin{array}{c}99.73 \\
(86.98)\end{array}$ & $\begin{array}{c}82.78 \\
(65.45)\end{array}$ \\
\hline FS 21 & $\begin{array}{c}77.22 \\
(61.48)\end{array}$ & $\begin{array}{c}93.33 \\
(75.00)\end{array}$ & $\begin{array}{c}80.56 \\
(63.81)\end{array}$ \\
\hline FO 22 & $\begin{array}{c}59.11 \\
(50.23)\end{array}$ & $\begin{array}{l}99.76 \\
(87.15)\end{array}$ & $\begin{array}{l}100.00 \\
(90.00)\end{array}$ \\
\hline FS 23 & $\begin{array}{c}55.22 \\
(47.97)\end{array}$ & $\begin{array}{l}95.56 \\
(77.80)\end{array}$ & $\begin{array}{c}84.67 \\
(66.92)\end{array}$ \\
\hline FS 24 & $\begin{array}{c}60.00 \\
(50.75)\end{array}$ & $\begin{array}{l}97.78 \\
(81.39)\end{array}$ & $\begin{array}{c}87.22 \\
(69.03)\end{array}$ \\
\hline FS 25 & $\begin{array}{c}68.89 \\
(56.09)\end{array}$ & $\begin{array}{c}99.73 \\
(86.98)\end{array}$ & $\begin{array}{c}91.67 \\
(73.19)\end{array}$ \\
\hline FO 26 & $\begin{array}{c}58.33 \\
(49.77)\end{array}$ & $\begin{array}{c}99.78 \\
(87.27)\end{array}$ & $\begin{array}{c}82.44 \\
(65.20)\end{array}$ \\
\hline FS 27 & $\begin{array}{c}47.78 \\
(43.71)\end{array}$ & $\begin{array}{c}99.73 \\
(86.98)\end{array}$ & $\begin{array}{c}85.00 \\
(67.19)\end{array}$ \\
\hline FS 28 & $\begin{array}{c}47.78 \\
(43.71)\end{array}$ & $\begin{array}{l}99.67 \\
(86.67)\end{array}$ & $\begin{array}{c}87.22 \\
(69.03)\end{array}$ \\
\hline FS 29 & $\begin{array}{c}75.00 \\
(59.98)\end{array}$ & $\begin{array}{c}99.73 \\
(86.98)\end{array}$ & $\begin{array}{c}89.44 \\
(71.01)\end{array}$ \\
\hline FO 30 & $\begin{array}{c}39.11 \\
(38.69)\end{array}$ & $\begin{array}{c}97.78 \\
(81.39)\end{array}$ & $\begin{array}{c}87.33 \\
(69.13)\end{array}$ \\
\hline FS 31 & $\begin{array}{c}58.33 \\
(49.77)\end{array}$ & $\begin{array}{c}93.33 \\
(75.00)\end{array}$ & $\begin{array}{c}85.00 \\
(67.19)\end{array}$ \\
\hline FS 32 & $\begin{array}{c}58.89 \\
(50.10)\end{array}$ & $\begin{array}{c}93.33 \\
(75.00)\end{array}$ & $\begin{array}{c}84.67 \\
(66.92)\end{array}$ \\
\hline FS 33 & $\begin{array}{c}78.33 \\
(63.23)\end{array}$ & $\begin{array}{c}82.22 \\
(65.03)\end{array}$ & $\begin{array}{c}89.78 \\
(71.34)\end{array}$ \\
\hline FS 34 & $\begin{array}{c}85.00 \\
(67.19)\end{array}$ & $\begin{array}{c}80.00 \\
(63.40)\end{array}$ & $\begin{array}{c}85.00 \\
(67.19)\end{array}$ \\
\hline FS 35 & $\begin{array}{c}74.44 \\
(59.61)\end{array}$ & $\begin{array}{c}82.22 \\
(65.03)\end{array}$ & $\begin{array}{c}86.89 \\
(68.74)\end{array}$ \\
\hline FS 36 & $\begin{array}{r}100.00 \\
(90.00)\end{array}$ & $\begin{array}{c}99.71 \\
(86.87)\end{array}$ & $\begin{array}{c}85.00 \\
(67.19) \\
\end{array}$ \\
\hline FS 37 & $\begin{array}{l}100.00 \\
(90.00)\end{array}$ & $\begin{array}{l}99.69 \\
(86.77)\end{array}$ & $\begin{array}{c}89.11 \\
(70.70)\end{array}$ \\
\hline FS 38 & $\begin{array}{r}100.00 \\
(90.00)\end{array}$ & $\begin{array}{c}80.00 \\
(63.40)\end{array}$ & $\begin{array}{c}89.11 \\
(70.70)\end{array}$ \\
\hline FS 39 & $\begin{array}{c}91.22 \\
(72.73)\end{array}$ & $\begin{array}{c}99.73 \\
(86.98)\end{array}$ & $\begin{array}{c}91.22 \\
(72.73)\end{array}$ \\
\hline FS 40 & $\begin{array}{c}90.56 \\
(72.08) \\
\end{array}$ & $\begin{array}{c}93.33 \\
(75.00)\end{array}$ & $\begin{array}{c}84.67 \\
(66.92)\end{array}$ \\
\hline FS 41 & $\begin{array}{c}52.22 \\
(46.25)\end{array}$ & $\begin{array}{c}80.00 \\
(63.40)\end{array}$ & $\begin{array}{c}89.44 \\
(71.02)\end{array}$ \\
\hline FS 42 & $\begin{array}{c}59.00 \\
(50.16)\end{array}$ & $\begin{array}{l}99.71 \\
(86.87)\end{array}$ & $\begin{array}{c}96.11 \\
(78.62)\end{array}$ \\
\hline FS 43 & $\begin{array}{c}45.78 \\
(42.55)\end{array}$ & $\begin{array}{c}99.73 \\
(86.98)\end{array}$ & $\begin{array}{c}95.78 \\
(78.11)\end{array}$ \\
\hline
\end{tabular}




\begin{tabular}{|c|c|c|c|}
\hline FS 44 & 56.11 & 99.71 & 97.89 \\
& $(48.49)$ & $(86.87)$ & $(81.61)$ \\
\hline FS 45 & 53.89 & 99.69 & 95.78 \\
& $(47.20)$ & $(86.77)$ & $(78.11)$ \\
\hline FS 46 & 54.44 & 81.11 & 93.56 \\
& $(47.53)$ & $(64.22)$ & $(75.26)$ \\
\hline FS 47 & 65.56 & 99.71 & $(62.00$ \\
& $(58.06)$ & $(86.87)$ & 84.67 \\
\hline FS 48 & 58.89 & 73.33 & $(66.92)$ \\
\hline FS 49 & $(50.10)$ & $(58.88)$ & 73.89 \\
& 100.00 & 82.22 & $(59.24)$ \\
\hline FS 50 & $(90.00)$ & $(65.03)$ & 80.56 \\
& 57.89 & 86.67 & $(63.81)$ \\
\hline FS 51 & $(49.52)$ & $(68.55)$ & 80.56 \\
& 67.22 & 80.00 & - \\
\hline Control & $(55.05)$ & $(63.40)$ & 85.57 \\
\hline Mean & - & - & 1.436 \\
\hline CD $(\mathbf{0 . 0 1})$ & 67.63 & 92.20 & $(1.199)$ \\
\hline SE.m \pm & 3.612 & 0.438 & 0.505 \\
& $(2.221)$ & $(0.321)$ & $(0.421)$ \\
\hline
\end{tabular}

Inoculated plants showed gradual increase in expression of wilt symptoms starting from 3rd day to fourth week. Pathogenic variability among the isolates was ascertained on the basis of the ability of each isolate to cause disease and the temporal variation in appearance of the specific symptoms viz., leaf chlorosis, yellowing and drooping, moderate wilting, severe wilting and death of plants. The appearance of the specific symptom varied depending on the virulence level of each isolate (Table 1). Based on pathogenicity reaction the isolates were categorized into 3 groups viz., highly pathogenic (isolates that caused complete wilting by seventh day), moderately pathogenic (isolates that caused complete wilting by 15 th day) and weakly pathogenic (isolates that caused chlorosis and moderate wilting even after 22nd day) (Table 2 ). All the three isolates of $F$. oxysporum (FO 22, FO 26 and FO 30) were highly pathogenic as the symptoms appeared in the most severe form and complete wilting and death of the plant was seen within first week after inoculation. Among 48 isolates of $F$. solani nineteen isolates (FS 1, FS 9 to FS 16, FS 19,
FS 21, FS 24, FS 27, FS 28, FS 47 to FS 51) were highly pathogenic. Twenty five isolates (FS 2 to FS 8, FS 17, FS 18, FS 20, FS 23, FS 25, FS 29, FS 31 to FS 38, FS 40, FS 43, FS 44, FS 46) were moderately pathogenic and four isolates (FS 39, FS 41, FS 42 and FS 45) were weakly pathogenic. The present results are also in conformity with the findings of Ferniah et al., (2014) who reported that the $F$. oxysporum isolate P1a was highly pathogenic that caused wilting in two chili cv. TM999 and Gantari with 40 and 63 per cent disease severity respectively. Similarly, Nirmaladevi and Srinivas (2012) identified 18 isolates of Fusarium oxysporum as highly pathogenic, 40 as moderately pathogenic and 20 as weakly pathogenic in their study in tomato cultivars. Raghu et al., (2016) also reported variations in pathogenicity of 36 isolates of Fusarium spp. on chilli. The pathogenic variability in Fusarium isolates might be brought about by either natural mutation, natural selection, genetic drift, gene flow or mating system (McDonald and Linde, 2002). The variation in the $F$. oxysporum isolates, in turn can occur by means of natural mutations, through 
parasexuality or heterokaryosis (Buxton, 1962; Kuhn et al., 1995).

\section{Sensitivity to fungicides}

Among the fungicides tested, irrespective of the isolates, maximum inhibition of growth was observed in carbendezim $(0.2 \%)$ followed by propiconazole $(0.05 \%)$, and least inhibition was observed in mancozeb $(0.25 \%)$. However, the isolates varied for their sensitivity to different fungicides. For carbendezim, majority of the isolates showed highest sensitivity with $80-90$ per cent inhibition of growth and isolate FS 48 was least sensitive with 73.83 per cent inhibition of growth. For mancozeb, nine isolates (FS 9, FS 10, FS 11, FS 30, FS 37, FS 38, FS 39, FS 40, FS 49) showed high sensitivity with 90100 per cent inhibition of growth, followed by isolates (FS 1 to FS 7, FS 13, FS 14, FS 15, FS 17 to FS 21, FS 24, FS 25, FS 29, FS 33 to FS 35, FS 47, FS 51) which showed 60-80 per cent inhibition of growth and 19 isolates (FS 8, FS 12, FS 16, FO 22, FO 23, FO 26, FS 27, FS 28, FS 30, FS 31, FS 41 to FS 46, FS 48, FS 50) showed least sensitivity with 45-60 per cent inhibition of growth. For propiconazole, four isolates (FS 14, FS 17, FS 18, FO 22, FS 42, FS to FS 46) showed highest sensitivity with $90-100$ per cent inhibition of growth and rest of the isolates showed 78-90 per cent inhibition of growth (Table 3). The isolates which showed least sensitivity might have developed resistance against those fungicides which needs to be considered while managing the disease. Sensitivity to fungicides may indicate usefulness of particular fungicide in management of the pathogen. Resistant individuals within in a fungal population may develop due to repeated application of fungicides (Eckert, 1988). In the present study the isolates collected from different locations varied for their sensitivity to different fungicides. Variations in sensitivity against fungicides in different Fusarium spp. and isolates have also been reported by Rajput $e t$ al., 2006; Amini et al., 2010; Andrabi et al., 2011; Maitlo et al., 2014; Dahal and Shrestha 2018.

\section{Acknowledgement}

The authors thank Department of Plant Pathology, Kittur Rani Channamma College of Horticulture, Arabhavi, University of Horticultural Sciences, Bagalkot, Karnataka, India India for providing all possible facilities for present study.

\section{References}

Amini, J. and Sidovich, D. F., 2010, The effects of fungicides on Fusarium oxysporum f. sp. lycopersici associated with fusarium wilt of tomato. J. Plant Prot. Res., 50(2): 172-178.

Andrabi, M., Vaid, A. and Razdan, V. K., 2011, Evaluation of different measures to control wilt causing pathogens in chickpea. J. Plant Prot. Res., 51(1): 5559.

Anonymous, 2005, Annual report of network project on wilt of chilli with special reference to cultural, morphological, molecular characterization and pathogenic variability of isolates of India, submitted to ICAR, New Delhi, p. 7.

Anonymous, 2017, National Horticulture Board, http;//www.nhb.gov.in.

Buxton, E.W., 1962, Parasexual recombination in banana-wilt Fusarium. Transact. British Mycol. Soc., 45: 274-279.

Dahal, N. and. Shrestha, R. K., 2018, Evaluation of efficacy of fungicides against Fusarium oxysporum f. sp. lentis in vitro at lamjung, Nepal. J. Inst. Agric. Anim. Sci., 35: 105-112.

Devika Rani, G. S., Naik, M. K., Raju, K. and 
Prasad, P. S., 2007, Prevalence of Wilt of Chilli and assessment of population dynamics of Fusarium in predominant Chilli (Capsicum annuum L.) growing regions of Karnataka. Soil Biol., 27(1\&2): 50-61.

Eckert, J.W., 1988. Historical development of fungicide resistance in plant pathogens. In: Delp, C.J. (Ed.), Fungicide resistance in North America. APS Press, St. Paul, pp.1-3.

Ferniah, R. S., Daryono, B. S., Kasiamdari, R. S. and Priyatmojo, A., 2014, Characterization and pathogenicity of Fusarium oxysporum as the causal agent of fusarium wilt in chilli (Capsicum annuum L.). J. Microbiol., 8(3): 121-126.

Kuhn, D.N., Cortes, B., Pinto, T. and Weaver, J. 1995. Parasexuality and heterokaryosis in Fusarium oxysporum f.sp.cubense. Phytopathol., 85: 11-19.

Leslie, J.F. and Summerell, B.A., 2006, The Fusarium Laboratory Manual. Blackwell Publishing Ltd.,Victoria, Australia, 388 pp.

Loganathan, M., Venkataravanappa, V., Saha, S., Sharma, B.K., Tirupathi, S. and Verma, M.K. 2013. Morphological, cultural and molecular characterizations of Fusarium wilt infecting tomato and chilli. In: National Symposium on Abiotic and Biotic Stress Management in Vegetable Crops, Indian Society of Vegetable Science, IIVR, Varanasi, April 12-14, 2013.
Maitlo, S. A., Syed, R.N., Rustamani, M. A., Khuhro, R. D., and Lodhi, A.M., 2014, comparative efficacy of different fungicides against fusarium wilt of chickpea (Cicer arietinum). Pak. J. Bot., 46(6): 2305-2312.

McDonald, B.A. and Linde, C., 2002, Pathogen population genetics, evolutionary potential and durable resistance. Ann. Rev. Phytopathol., 40: 439-479.

Nirmaladevi, D., and Srinivas, C., 2012, Cultural, Morphological, and Pathogenicity Variation in Fusarium oxysporum f. sp. lycopersici Causing Wilt of Tomato. Sci. Rep., 6: 21367213680.

Raghu, S., 2014, Studies on chilli wilt complex disease. Ph.D. Thesis, University of Agricultural Sciences, Dharwad.

Raghu, S., Benagi, V. I. and Nargund, V. B., 2016, Cultural, morphological and pathogenic variability among the isolates of Fusarium solani causing wilt disease of chilli. Int. J. of Agri. Sci. and Res., 4:6 96-105.

Rajput, A. Q., Arain, M. H., Pathan, M. A., Jskani, V and Lodhi, A. M., 2006, Efficacy of different fungicides against fusarium wilt of cotton caused by Fusarium oxysporum f. Sp. vasinfectum. Pak. J. Bot., 38(3): 875880.

\section{How to cite this article:}

Deepthi Gowda, S., S. Amruta Bhat, V. Kantharaju, Sandhyarani Nishani, Abdul Kareem and Hadimani, H. P. 2020. Pathogenic Variability and Fungicidal Sensitivity of Fusarium spp. Causing Wilt in Chilli in Karnataka. Int.J.Curr.Microbiol.App.Sci. 9(10): 301-309. doi: https://doi.org/10.20546/ijcmas.2020.910.038 